Journal of Animal and Veterinary Advances 10 (15): 1938-1946, 2011

ISSN: $1680-5593$

(C) Medwell Journals, 2011

\title{
Lepidoptera Fauna in Artvin Province in North Black Sea Region of Turkey
}

\author{
Temel Gokturk \\ Department of Forest Entomology and Protection, Faculty of Forestry, \\ Artvin Coruh University, Artvin, Turkey
}

\begin{abstract}
The Lepidopteran species of Artvin province were investigated. To collect specimens, a sweep net and light traps were used. The butterfly species that were captured in Artvin centrum (Kafkasor, Sacinka, Melodagi, Seyitler), Ardanuc (Kutul, Meseli, Fidanlik), Arhavi, Hatila National park, Hopa (Cankurtaran, Kemalpasa), Murgul, Savsat (Sahara, Karagol), Yusufeli (Barhal, Yaylalar, Altiparmak, Kilickaya). In the end of the study, a total of 192 species belonging to 15 families of the order Lepidoptera were identified. The family Lycaenidae was represented by the highest number of species (62) followed by Satryridae (27), Nymphalidae (25) and Hesperiidae (20). Most of the species found in this study were captured in Yusufeli.
\end{abstract}

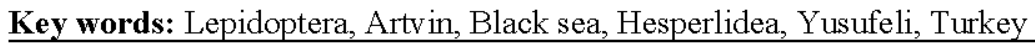

\section{INTRODUCTION}

There are well over 1 million known species of insects in the world and some experts estimate that there might be as many as 10 million. Lepidoptera is a very large order that includes some of the most beautiful species and some of the most economically important pests in the class Insecta (Pedigo, 1996). This order is recognized as one of the largest order of insects (Romoser and Stoffolano, 1994) having $>150,000$ described species (Groombridge, 1992). World butterflies number about 17,280 species representing described taxa that have not been synonymised and are currently grouped into 1855 genera, 35 subfamilies and 7 families (Shields, 1989). Besides, Cassie (2007) has declared that there are 17,000 species of butterflies in the world.

There is an increasing body of evidence suggesting that connectivity and quality of habitats in agricultural landscapes have a significant effect on survival of animal species including arthropods (Andow, 1991; Rossing et al., 2003). Lepidoptera are found in a wide variety of habitats but are almost always associated with higher plants especially angiosperms. Butterflies are very important ecological indicators since, the majority of butterflies are sensative to various environmental changes and their number in most cases is directly proportional to the ecological state of areas inhabited by them they are considered very important ecological indicators (Borror et al., 1989; Gillott, 2005). Butterflies are most active during the warmer months of the year, mid-spring to early autumn.

Past studies have dealt primarily with taxa identification. There are several regional studies to determine the Lepidoptera fauna in Turkey but not completed and remain unknown mostly regional areas (Akbulut et al., 2003; Beskardes, 2002; Can, 2008; Hakyemez, 1994; Kansu, 1963; Kaygin et al., 2009; Mol and Avci, 1997; Mol, 1977; Okyar and Aktac, 1999, 2006).

Artvin has a rather rich fauna because of numeraous microclimas created by climatic features and forms. However, the fauna of the Artvin has still not been fully studied. This study was carried out to determine the Lepidopteran species of Artvin province. Researchers found some Lepidopteran species in Artvin earlier however, this study is important since, it is the first and comprehensive study of Lepidopteran species.

\section{MATERIALS AND METHODS}

The main material of this study has been the species of Lepidoptera existing in Artvin province, Turkey. Artvin is located in eastern part of Black sea region of Turkey. As a Black sea region province, Artvin which has a total of $7,367 \mathrm{~km}^{2}$ land area is in the West of Ardahan, North of Erzurum, East of Rize and West of Georgia. Artvin includes Camili biosphere reserve known as the first biosphere site of Turkey is one of the 507 sites in 102 countries worldwide. In the province although, Arhavi and Hopa's alluvial plains, the high plateaus possess a big share of the land mass. As a typical Black sea region province in every season a rainfall climate is seen in the coastal areas of the province while in the interior sides a continental climate, cousisting of harsher winters and less summer precipitation is seen. The altitude of the area ranges from $100-3925 \mathrm{~m}$.

The dendroflora of the Artvin district is represented by 171 taxa, 119 species, 33 sub-species and 19 varieties belonging to 81 genera and 42 families. Gymnosperms and 
Angiosperms comprised 8 and 163 taxa, respectively. Pseudomacchie, forest, alpine, subalpine, rocky and hydrophytic are the main vegetation types in Artvin. Forest vegetation is the most widespread in the study area (Eminagaoglu and Bak, 2008).

The study area included 8 locaties in Artvin (Table 1). The adult butterflies were collected mainly from herbaceous plants in different habitats such as open habitats, road sides, agricultural fields, meadows vineyards and forest where they were active between April and October in 2007 and 2009.

Butterflies caught with a sweep net were killed in potassium cyanide or ethyl acetate and were then transferred to the laboratory in a fatty envelope. At night,

Table 1: Geographic lacality coordinatescof collected specimens

\begin{tabular}{lll} 
& \multicolumn{2}{l}{ Locality coordinates } \\
& ---------- \\
Locality & North (N) & South (E) \\
\hline Artvin central & $41^{\circ} 10^{\prime} 00^{\prime \prime}$ & $41^{\circ} 48^{\prime} 58^{\prime \prime}$ \\
(Kafkasor, Sacinka, Melodagi, Seyitler) & & \\
Ardanuc (Kutul, Meseli, Fidanlik) & $41^{\circ} 07^{\prime} 42^{\prime \prime}$ & $42^{\circ} 02^{\prime} 32^{\prime \prime}$ \\
Arhavi & $41^{\circ} 21^{\prime} 07^{\prime \prime}$ & $41^{\circ} 18^{\prime} 41^{\prime \prime}$ \\
Hatila National park & $41^{\circ} 11^{\prime} 11^{\prime \prime}$ & $41^{\circ} 44^{\prime} 09^{\prime \prime}$ \\
Hopa (Cankurtaran, Kemalpasa) & $41^{\circ} 23^{\prime} 13^{\prime \prime}$ & $41^{\circ} 26^{\prime} 12^{\prime \prime}$ \\
Murgul & $41^{\circ} 16^{\prime} 28^{\prime \prime}$ & $41^{\circ} 33^{\prime} 58^{\prime \prime}$ \\
Savsat (Sahara, Karagol) & $41^{\circ} 4140^{\prime \prime}$ & $41^{\circ} 21^{\prime} 51^{\prime \prime}$ \\
Yusufeli & $40^{\circ} 4932^{\prime \prime}$ & $41^{\circ} 32^{\prime} 55^{\prime \prime}$ \\
(Barhal, Yaylalar, Altiparmak, Kilickaya) & & \\
\hline
\end{tabular}

nocturnal specimens were collected by light traps. Specimens were pinned using insect pins (mostly, 3-5 sized) and were mounted on spreading boards. Collection and preservation techniques used in this research were based mainly on Canakcioglu (1993). In adition to these methods confining in polyester was used. Each lepidopteran specimen was identified with the aid of stereomicroscope. A variety of literature sources were used for identification (Covell, 1984; Higgins, 1982). The identifacation of species was performed according to Hesselbarth. Faunistic data of all the species collected in the present study are shown (Table 1 and Fig. 1). Family names and species are listed according to Siberian Zoological Museum List. The specimens are stored at the collection room of Forest Entomology and Protection Unit, Forest Faculty, Artvin Coruh University, Artvin, Turkey.

\section{RESULTS AND DISCUSSION}

During the 3 years, total 192 secimens were collected. A total of 349 Lepidoptera specimens were collected in Artvin province. A total of 192 species belonging to 15 families of the order Lepidoptera were identified and are listed as follows. The samples mostly identified have been Lycaenidae, Noctuidae, Nymphalidae and Satyridae. The

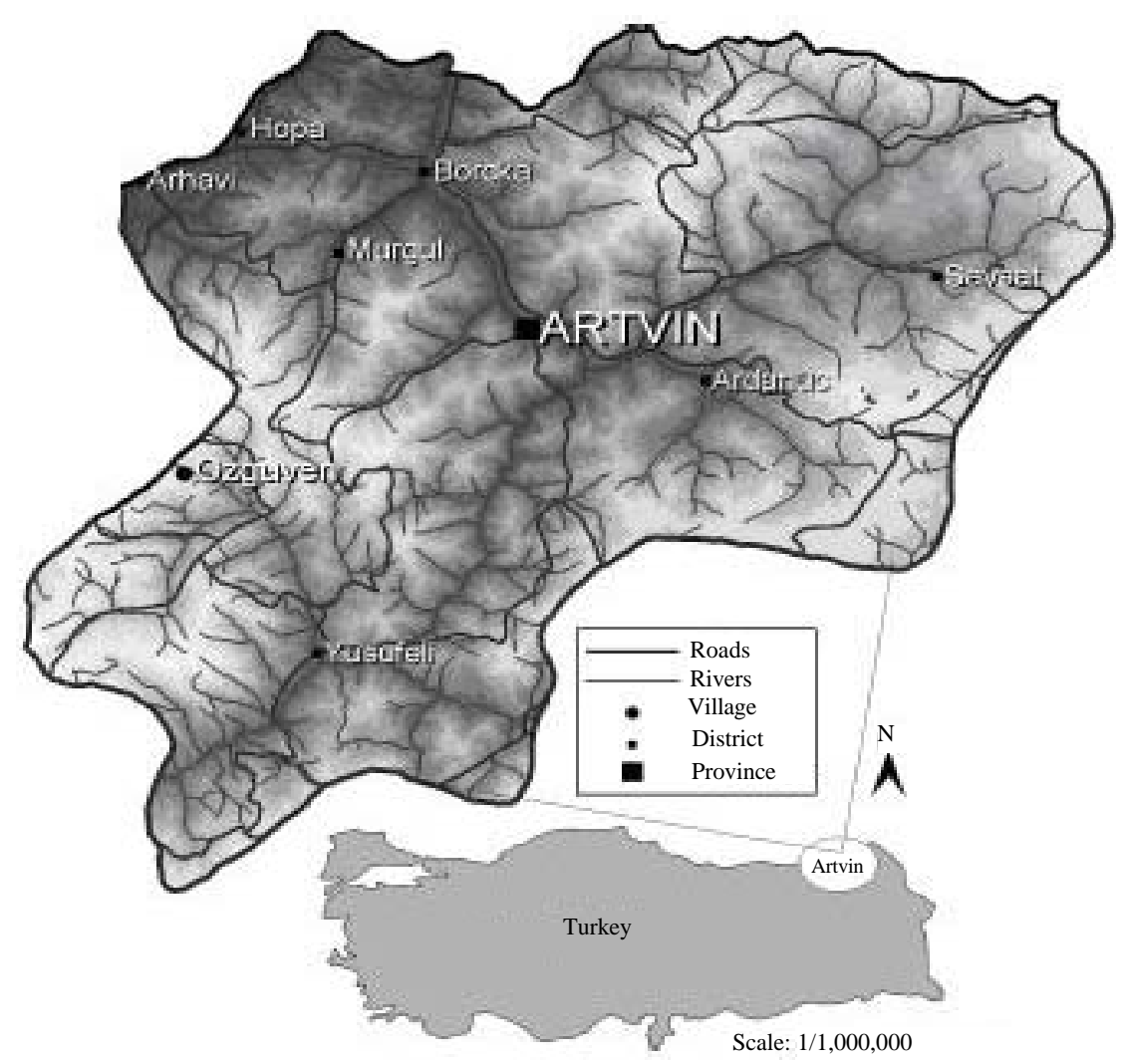

Fig. 1: Study area 
Table 2: Identification species and localities in artvin province

Order Lepidoptera

Suborder rhopalocera

Superfamily hesperioidea Latreille (1809)

Family hesperiidae Latreille (1809)

Subfamily Hesperiinae Latreille (1809)

Thymelicus lineola (Ochsenheimer, 1808) (26.07.2007, Yusufeli Yaylalar; 27.07.2009 Yusufeli Barhal; 22.06.2008, Ardanuc Kutul)

Thymelicus sylvestris (Poda, 1761) (17.08.2008, Yusufeli Kilickaya; 27.07.2009, Yusufeli Barhal)

Thymelicus novus (Reverdin, 1916 (23.07.2008, Yusufeli Barhal; 14.08.2009, Yusufeli Yaylalar)

Hesperia comma (Linnaeus, 1758) (20.07.2007 and 10.08.2008, HatilaNP; 22.07.2008, Ardanuc)

Ochlodes venotus (Bremer and Grey, 1852) (28.06.2007, Ardanuc; 14.08.2009, Yusufeli Barhal)

Sub-family Pryginae Burmeister (1878)

Erynnis tages (Linnaeus, 1758) (22.07.2008, Ardanuc; 27.07.2009, Yusufeli)

Erynnis marloyi (Boisduval, 1834) (07.07.2007, Ardanuc; 06.08.2008, Ardanuc)

Carcharodus alceae (Esper, 1780) (29.06.2009 and 23.07.2008, Yusufeli Yaylalar)

Carcharodus lavatherae (Esper, 1783) (18.07.2008, Hatila NP)

Carcharodus flocciferus (Zeller, 1847) (18.07.2008, Hatila NP)

Carcharodus orientalis (Reverdin, 1913) (15.08.2009, Yusufeli Yaylalar)

Spialia phlomidis (Herrich-Schaffer, 1845) (23.06.2007 and 15.07.2008, Murgul)

Spialia orbifer (Hubner, 1823) (23.06.2007, Murgul; 27.07.2009, Yusufeli Altiparmak)

Pyrgus melotis (Duponchel, 1834) (22.06.2009, Aradanuc)

Pyrgus jupei (Alberti, 1967) (22.06.2009, Ardanuc; 18.07.2007, Murgul)

Pyrgus serratulae (Rambur, 1839) (13.06.2008, Ardanuc; 19.07.2009, Hatila NP)

Pyrgus sidae (Esper, 1784) (23.07.2007, Savsat Sahara)

Pyrgus cinarce (Rambur, 1839) (23.07.2007, Savsat Sahara)

Pyrgus alveus (Hubner, 1803) (22.06.2009, Ardanuc)

Pyrgus armoricanus (Oberthur, 1910) (27.07.2009, Yusufeli Yaylalar; 21.06.2009, Artvin Sacinka)

Superfamily Papilionoidae Latreille (1802)

Family Papilionidae Latreille (1802)

Subfamily Papilioninae Latreille (1802)

Parnassius mnemosyne (Linnaeus, 1758) (29.06.2009, Yusufeli Kilickaya)

Pornassius nortmanni (Siemaschko, 1850) (12.06.2008, Artvin Sacinka)

Parnassius apollo (Linnaeus, 1758) (19.05.2009 and 12.06.2008, Artvin Seyitler; 11.06.2008, Hatila NP.; 13.06.2009, Ardanuc)

Iphiclides podalirius (Linnaeus, 1758) (04.07.2007, Artvin; 12.07.2007, Hatila NP; 27.07.2008, Yusufeli Kilickaya)

Pcpilio machaon (Linnaeus, 1758) (29.06.2009, Yusufeli Barhal; 22.06.2009, Ardanuc Fidanlik; 25.06.2007 and 20.06.2009, Hatila NP)

Family Pieridae Duponchel (1835)

Sub-family, Dismorphiinae Schatz (1886)

Leptidea sinapis (Linnaeus, 1758) (08.06.2008, Arhavi; 29.06.2009, Yusufeli; 16.06.2009, Murgul; 04.07.2007, Artvin; 07.07.2007, Ardanuc Meseli; 12 07.2007 , Hatila NP)

Leptidea duponcheli (Staudinger, 1871) (22.06.2009, Ardanuc Kutul)

Sub-family, Pierinae Duponchel (1835)

Anthochoris cordomines (Linnaeus, 1758) (12.06.2008, Artvin Sacinka; 20.06.2009, Hatila NP.; 05.07.2007, Artvin Seyitler; 10.07.2007, Savsat; 08.06.2008, Arhavi; 15.06.2009, Hopa)

Aporia crataegi (Linnaeus, 1758) (17.05.2007 and 12.06.2008, Artvin; 29.06.2009, Yusufeli Yaylalar; 23.07.2008 and 27.07.2009, Yusufeli Barhal; 23.06.2007, Murgul; 08.06.2008, Arhavi; 15.06.2009, Hopa)

Pontia edusa (Fabricius, 1777) (24.05.2008, Ardanuc; 30.06.2007, Yusufeli; 12.06.2008, Artvin Sacinka; 20.06.2009, Hatila NP)

Pontia chloridice (Hubner, 1813) (23.07.208, Yusufeli Yaylalar; 27.07.2009, Yusufeli Kilickaya)

Pieris napi (Linnaeus, 1758) (22.06.2007, Hopa; 05.07.2007 Artvin Seyitler; 11.07.2007 and 20.07.2007, Savsat; 08.06.2008, Arhavi; 22.07.2008, Ardanuc) Pieris bryonice (Hubner, 1806) (24.07.2008, Ardanuc; 20.07.2008, Savsat)

Pieris monnii (Mayer, 1851) (23.06.2007, Murgul; 15.06.2009, Hopa; 14.06.2008 Savsat)

Pieris ergane (Geyer, 1828) (13.06.2008, Ardanuc; 12.06.2008, Artvin Sacinka)

Pieris rapae (Linnaeus, 1758) (12.06.2008, 04.07.2007, 11.08.2008 and 19.06.2009, Artvin Sacinka; 07.07.2007 and 22.06.2009, Ardanuc; 10.07.2007, Savsat; 08.06.2008, Arhavi; 08.06.2008, Hopa)

Pieris brassicae (Linnaeus,1758) (22.05.2008 and 18.06.2008, Artin Seyitler; 07.07.2007 and 13.06.2008, Ardanuc; 15.06.2009, Arhavi; 08.06.2008, Hopa; 14.06.2008, Savsat; 30.06.2007, Yusufeli; 12.07.2007, Hatila NP)

Sub-family Coliadinae Swainson (1827)

Colias alfacoriensis (Ribbe, 1905) (22.07.2008, Ardanuc; 19.07.2007, Artvin Sacinka)

Colias crocea (Fourcroy, 1785) (04.04.2007, Artvin Seyitler, 07.07.2007 and 22.07.2009 Ardanuc; 22.06.2008, Arhavi; 23.07.2008, Yusufeli Barhal; 03.07.2008, Artvin; 20.07.2008, Savsat; 13.07.2009, Hopa Kemalpasa)

Colias caucasica (Staudinger, 1871) (23.07.2008, Yusufeli Yaylalar)

Gonepteryx rhamni (Linnaeus, 1758) (18.07.2007, Murgul; 27.07.2009, Yusufeli Yaylalar)

Gonepteryx forinosa (Zeller, 1847) (23.07.2008, Yusufeli Kilickaya)

Family Lycaenidae Leach (1815)

Sub-family Theclinae Swains on (1831)

Thecla betulae (Linnaeus, 1758) (27.07.2009, Yusufeli)

Satyrium spini (Denis and Schiffermüller, 1775) (12.06.2008, Artvin Sacinka)

Satyrium ilicis (Esper, 1779) (19.06.2009, Artvin Kafkasor)

Satyrium acaciae (Fabricius, 1787) (23.07.2008, Yusufeli Barhal)

Satyrium abdominalis (Gerhard, 1850) (23.07.2008, Yusufeli Barhal)

Satyrium w-album (Knoch, 1782) (22.07.2008, Ardanuc; 27.07.2009, Yusufeli Kilickaya; 17.07.2008, Artvin Sacinka; 26.07.2007, Yusufeli Barhal)

Favonius quercus (Linnaeus, 1758) (17.07.2008, Artvin Sacinka) 
Table 2: Countined

Sub-family Lycaeninae Leach (1815)

Lycaena phlaeas (Linnaeus, 1761) (28.06.2007, Ardanuc Kutul)

Lycaena virgaureae (Linnaeus, 1758) (18.06.2009, Artvin Kafkasor; 23.07.2008, Yusufeli Yaylalar)

Lycaena tityrus (Poda, 1761) (30.07.2009, Yusufeli)

Lycaena alciphron (Rottemburg, 1775) (29.07.2007, Yusufeli Kilickaya)

Lycaena candens (Herrich-Schaffer, 1844) (27.07.2009, Yusufeli Yaylalar)

Lycaena thetis (Klug, 1834) (15.08.2009, Yusufeli)

Lycaena asabinus (Herrich and Schaffer, 1851) (14.07.2009, Murgul)

Lycaena thersamon (Esper, 1784) (17.08.2008, Yusufeli Yaylalar)

Lycaena ochimus (Herrich and Schaffer, 1851) (27.07.2009, Yusufeli Kilickaya; 15.08.2009 Yusufeli Barhal)

Sub-family Polyommatinae Swainson (1827)

Cupido argiades (Pallas, 1771) (14.08.2007, Yusufeli; 22.07.2008, Ardanuc Kutul)

Cupido osiris (Meigen, 1829) (20.07.2008, Savsat; 17.07.2008, Artvin Sacinka)

Turanana endymion (Freyer, 1850) (23.07.2008, Yusufeli)

Pseudophilotes vicrama (Moore, 1865) (27.07.2007, Yusufeli Kilickay a; 23.07.2008, Yusufeli Yaylalar)

Glaucopsyche alcon (Denis and Schiffermuller, 1775) (17.07.2009, Artvin Kafkasor)

Plebejides pylaon (Fischer and Waldheim, 1832) (23.07.2008, Yusufeli)

Plebejus argus (Linnaeus, 1758) (30.07.2009, Yusufeli)

Plebejus argyrognomon (Bergstrasser, 1779) (27.07.2009, Yusufeli; 23.07.2009, Savsat Karagol)

Plebejus idas (Linnaeus, 1761) (29.06.2009, Yusufeli)

Plebejus eurypilus (Freyer, 1851) (29.06.2009, Yusufeli)

Plebejus pyrencicus (Boisduval, 1840) (25.07.2008, Yusufeli)

Plebejus madicus (Verity, 1935) (23.07.2008, Yusufeli Yaylalar)

Plebejus eumedon (Esper, 1780) (23.07.2008, Yusufeli Yaylalar; 20.07.2008, Savsat Sahara; 22.07.2009, Ardanuc; 19.07.2009 Hatila NP)

Plebejus agestis (Denis and Schiffermuller, 1775) (26.07.2007, Yusufeli)

Plebejus artaxerxes (Fabricius, 1793) (2.06.2009, Ardanuc; 15.06.2009, Hopa)

Plebejus anteros (Freyer, 1838) (4.08.2008, Yusufeli)

Polyommatus eros (Ochsenheimer, 1808) (4.08.2008, Yusufeli)

Polyommatus loewii (Zeller, 1847) (02.08.2007, Murgul)

Polyommatus artvinensis (Carbonell, 1997) (29.07.2009, Yusufeli)

Polyommatus semiargus (Rottemburg, 1775) (29.07.2009, Yusufeli)

Polyommatus coelestinus (Eversmann, 1848) (27.07.2009, Yusufeli Kilickaya)

Polyommatus dorylas (Jermyn, 1827) (24.07.2008, Yusufeli, Kilickaya)

Polyommatus eumedon (Esper, 1780) (23.07.2008, Yusufeli)

Polyommatus forsteri (Pfeiffer, 1938) (18.07.2008, Hatila NP.)

Polyommatus pyrenaicus (Freyer, 1845) (16.08.2009, Yusufeli)

Polyommatus amondus (Schneider, 1792) (16.08.2009, Yusufeli)

Polyommatus thersites (Cantener, 1834) (29.06.2009, Yusufeli Yaylalar)

Polyommatus aedon (Christoph, 1887) (11.08.2009, Ardanuc)

Polyommatus iccorus (Rottemburg, 1775) (11.08.2009, Ardanuc; 07.08.2007, Savsat Sahara)

Polyommatus daphnis (Denis and Schiffermuller, 1776) (16.08.2009, Yusufeli)

Polyommatus bellorgus (Rottemburg, 1775) (16.08.2009, Yusufeli)

Polyommatus corydonius (Herrich-Schaffer, 1852) (21.07.2009, Ardanuc, 27.07.2009, Yusufeli Yaylalar)

Polyommatus ripartii (Freyer, 1830) (28.07.2009, Yusufeli)

Polyommatus aserbeidschonus (Forster, 1956) (28.07.2009, Yusufeli)

Polyommatus huberti (Carbonell, 1993) (14.08.2007, Yusufeli)

Polyommatus nince (Forster, 1956) (24.07.2008, Yusufeli Kilickaya)

Polyommatus admetus (Esper, 1783) (29.07.2009, Yusufeli Kilickaya)

Polyommatus hopfferi (Gerhard, 1851) (20.07.2008, Savsat; 25.07.2007, Ardanuc; 27.07.2009, Yusufeli)

Polyommatus merhaba (Prins et al.,1991) (28.07.2009, Yusufeli)

Polyommatus damon (Denis and Schiffermuller, 1775) (27.07.2009, Yusufeli Yaylalar, 14.08.2007, Yusufeli Barhal)

Polyommatus iphigenia (Herrich-Schiffer,1847) (28.07.2009, Yusufeli)

Polyommatus mithridates (Tosoet Balletto, 1976) (25.07.2008, Yusufeli)

Polyommatus turcicus (Kocak, 1977) (25.07.2008, Yusufeli)

Celastrina orgiolus (Linnaeus, 1758) (22.07.2008, Ardanuc)

Lampides boeticus (Linnaeus, 1767) (24.07.2007, Savsat Sahara)

Family Satryridae Boisdual (1833)

Sub-family Satyrinae Boisdual (1833)

Coenonympha leander (Esper, 1784) (20.06.2009, Hatila NP.)

Cononympha pamphilus (Linnaeus, 1758) (20.06.2009, Hatila NP.)

Coenonympha symphita (Lederer, 1870) (14.07.2009, Murgul,)

Erebia medusa (Denis and Schiffermuller, 1775) (19.07.2008, Artvin Sacinka; 21.07.2007, Artvin Kafkasor; 25.07.2009, Ardanuc; 24.07.2008, Yusufeli)

Erebia aethiops (Esper, 1777) (25.07.2009, Ardanuc Meseli; 21.07.2007, Artvin Kafkasor)

Erebia hewitsonii (Lederer, 1864) (22.06.2007, Hopa; 06.07.2008, Artvin; 25.07.2008, Yusufeli)

Erebia melancholica (Herrich and Schaffer, 1850) (06.07.2008, Artvin Kafkasor)

Erebia grancasica (Jachontov, 1909) (28.07.2009, Yusufeli)

Hyponephele lycaon (Rottemburg, 1775) (28.07.2009, Yusufeli)

Hyponephele lupina (Costa, 1836) (25.07.2009, Ardanuc Kutul)

Hipparchia syriaca (Staudinger, 1871) (26.07.2008, Yusufeli Barhal)

Hipparchia statilinus (Hufnagel, 1766) (27.07.2009, Yusufeli) 
Table 2: Couuntined

Hipparchia parisatis (Kollar, 1849) (22.07.2008, Ardanuc Kutul)

Brintesia circe (Fabricius, 1775) (24.07.2008, Yusufeli)

Arethusana arethusa (Denis and Schiffermuller, 1775) (07.08.2007, Savsat)

Satyrus amasinus (Staudinger, 1861) (07.08.2007, Savsat; 09.08.2007, Yusufeli)

Pseudochazara geyeri (Herrich and Schaffer, 1846) (28.07.2009, Yusufeli Altiparmak)

Pseudochazara mniszechii (Herrich and Schaffer, 1851) (28.07.2009, Yusufeli Altiparmak)

Chazara briseis (Linnaeus, 1764) (29.07.2009, Yusufeli Kilickaya)

Chazara bischoffii (Herrich and Schoffer, 1846) (29.07.2009, Yusufeli Kilickaya)

Melanargiagalathea (Linnaeus, 1758) (25.07.2008, Yusufeli Yaylalar)

Melanargia larissa (Geyer, 1828) (24.07.2008, Yusufeli Yaylalar)

Moniola jurtina (Linnaeus, 1758) (04.07.2007, Artvin; 07.07.2007, Ardanuc; 12.07.2007 Hatila NP.; 01.07.2008, Yusufeli; 23.07.2008, Yusufeli Barhal) Sub-family Elymniinae (Herrich and Schaffer, 1864)

Lasiommata megera (Linnaeus, 1767) (19.07.2009, Hatila NP.; 29.07.2008, Yusufeli Kilickaya)

Lasiommata petropolitana (Fabricius, 1787) (26.07.2007, Yusufeli, 19.07.2009, Hatila NP.)

Lasiommata maera (Linnaeus, 1758) (26.07.2007, Yusufeli; 24.07.2007, Savsat Sahara)

Pararge aegeria (Linnaeus, 1758) (05.07.2007 Artvin; 07.07.2007 Ardanuc; 20.07.2008, Savsat )

Family Nymphalidae Swainson (1827)

Sub-family Apaturinae (Boisduval, 1840)

Thaleropis ionia (Fischer and Eversmann, 1851) (04.07.2009, Artvin Sacinka)

Sub-family Limenitidinae (Behr, 1864)

Limenitis reducta (Staudinger, 1901) (25.07.2008, Yusufeli Yaylalar)

Sub-family Nymphalinae (Swainson, 1827)

Polygonia c-album (Linnaeus, 1758) (28.07.2009, Yusufeli Barhal)

Polygonia egea (Cramer, 1775) (26.07.2007, Yusufeli Yaylalar)

Vanessa atalonta (Linnaeus, 1758) (25.06.2007 and 19.07.2009, Hatila NP., 02.08.2007 Murgul; 06.08.2007 Ardanuc; 23.06.2009 Savsat Sahara; 17.08.2009 Yusufeli Barhal)

Vonessa cardui (Linnaeus, 1758) (07.07.2007 and 06.08.2007 Ardanuc; 12.07.2007, Hatila NP.; 02.08.2007 Murgul; 19.07.2009, Hatila NP.)

Aglais urticae (Linnaeus, 1758) (17.08.2008, Yusufeli; 06.08.2007, Ardanuc)

Sub-family Argynninae (Fabricius, 1807)

Melitcea phoebe (Oberthür, 1876) (09.08.2007, Yusufeli)

Melitae a trivia (Denis and Schiffermueller, 1775) (27.09.2009, Yusufeli)

Melitcea didyma (Esper, 1779) (24.07.2007, Savsat)

Melitcea interrupta (Kolenati, 1846) (24.07.2007, Savsat)

Melitce a diamina (Lang, 1789) (25.07.2008, Yusufeli Yaylalar)

Melitaea athalia (Rottemburg, 1775) (28.07.2007, Yusufeli)

Melitae a cinxia (Linnaeus, 1758) (29.07.2007, Yusufeli Kilickaya)

Boloria caucasica (Staudinger, 1861) (28.07.2009, Yusufeli Altiparma; 07.07.2008, Artvin Melodagy)

Boloria euphrosyne (Linnaeus, 1758) (28.07.2007, Yusufeli)

Brenthis dophne (Bergstrasser, 1780) (23.07.2008, Yusufeli)

Brenthis hecate (Denis and Schiffermuller, 1775) (27.07.2009, Yusufeli Barhal)

Issoria lathonia (Linnaeus, 1758) (05.07.2007, Artvin; 12.07.2007, Hatila NP.; 14.07.2008, Artvin Kafkasor; 22.06.2007, Hopa Cankurtaran)

Argynnis pophia (Linnaeus, 1758) (12.07.2007, Hatila NP.; 11.08.2008, Artvin; 02.08.2008, Artvin Kafkasor; 10.07.2009, Artvin)

Argynnis pandora (Denis and Schiffermuller,1775) [02.08.2008, Artvin Kafkasor)

Argynnis aglaja (Linnaeus, 1758) (19.07.2009, Hatila NP.; 18.07.2007 Murgul; 06.08.2007 Ardanuc)

Argynnis adippe (Denis and Schiffermuller, 1775) (19.07.2009, Hatila NP.; 07.08.2007, Savsat Karagol)

Argynnis niobe (Linnaeus, 1758) (04.08.2007, Hatila NP.)

Euphydryas aurinia (Rottemburg, 1775) (04.08.2008, Artvin Kafkasor)

Sub-order Heterocera

Superfamily Zygaenoidea Latreille (1809)

Family Zygaenidae Latreille (1809)

Sub-family Zygaeninae Latreille (1809)

Zygaena filipendulce (Linnaeus, 1758) (25.06.2007, Hatila NP.; 15.07.2007, Artvin Sacinka)

Super family Bombycoidea Latreille (1803)

Family Saturniidae Boisduval (1837)

Sub-family Saturniinae Boisduval (1837)

Saturnia pyri (Denis and Schiffermuller, 1775) (10.06.208 and 11.06.2009, Artvin Sey itler; 28.06.2007, Ardanuc Fidanlik; 13.07.2008, Arhavi; 13.07.2009,

Hopa Kemalpasa)

Super family Geometroidea Leach (1815)

Family Geometridae Leach (1815)

Sub-family Sterrhinae Meyrick (1892)

Scopula rubiginata (Hufnagel, 1767) (23.06.2007, Murgul; 17.06.2009, Artvin Kafkasor)

Sub-family Lar entiinae (Dup onchel, 1845)

Hydriomena impliwiata (Denis and Schiffermuller, 1775) (20.06.2009, Hatila NP.)

Sub-family Geometrinae Leach (1815)

Phaiogramma etruscaria (Zeller, 1849) (16.06.2009, Murgul; 25.06.2007, Hatila NP.)

Sub-family Ennominae Leach (1815)

Alcis repandata (Linnaeus, 1758) (08.07.2008, Artvin Sacinka)

Compaea margaritata (Linnaeus, 1767) (19.07.2009, Hatila NP.)

Peribatodes rhomboidaria (Denis and Schiffermuller, 1775) (28.06.2007, Ardanuc)

Opisthograptis luteolata (Linnaeus, 1758) (03.07.2008, Artvin Kafkasor) 
Table 2: Countined

Biston betulorius (Linnaeus, 1758) (13.07.2009, Hopa; 13.07.2009, Arhavi)

Superfamily Sphingoidea Latreille (1802)

Family Sphingidae Latreille (1802)

Sub-family Sphinginae Latreille (1802)

Agrius convolvuli (Linnaeus, 1758) (03.07.2008, Artvin Kafkasor; 18.07.2008, Hatila NP.)

Laothoe populi (Linnaeus, 1758) (06.07.2009, Artvin Kafkasor; 14.07.2008, Hatila NP.)

Sub-family Macroglossinae Haris (1839)

Mocroglossum stellatorum (Hufnagel, 1766) (23.06.2007, Murgul)

Superfamily Noctuoidea Latr eille (1809)

Family Arctiidae Leach (1815)

Sub-family Arctiinae Leach (1815)

Arctia villica (Linnaeus, 1758) (02.07.2009, Artvin Kafkasor; 07.07.2007, Ardanuc; 12.07.2007 and 18.07.2008, Hatila NP.)

Callimorpha quadripunctaria (Poda, 1761) (02.07.2009, Artvin Kafkasor, 2.07.2007, Hatila NP.)

Hyphantria cunea (Drury, 1773) (22.06.2008 and 13.07.2009, Hopa Kemalpasa)

Sub-family Syntominae Linnaeus (1758)

Amata ragazzii (Turati, 1917) (16.06.2009, Murgul)

Amata phegea (Linnaeus, 1758) (16.06.2009, Murgul)

Family Noctuidae Latreille (1809)

Sub-family Plusiinae Boisduval (1828)

Autogropha gamma (Linnaeus, 1758) (02.07.2009, Artvin Kafkasor; 14.07.2008, Hatila NP.)

Sub-family Catocalinae Boisduval (1828)

Cotocala elocata (Esper, 1787) (25.06.2007, Hatila NP.)

Catocala nupta (Linnaeus, 1767) (20.06.2009, Hatila NP.)

Sub-family Noctuinae Latreille (1809)

Agrotis c-nigrum (Linnaeus, 1758) (09.06.2008, Murgul)

Agrotis saucia (Hubner, 1808) (09.06.2008, Murgul)

Ammoconia caecimacula (Denis and Schiffermuller, 1775) (07.06.2008, Artvin Kafkasor)

Sub-family Hadeninae Guenée (1837)

Mamestra oleracea (Linnaeus, 1758) (10.06.2008, Artvin Kafkasor, 17.06.2009, Artvin Sacinka)

Mythimna l-album (Linnaeus, 1767) (10.06.2008, Artvin Kafkasor; 17.06.2009, Artvin Sacinka)

Mythimna ferrago (Fabricius, 1787) (06.07.2008, Artvin Kafkasor; 24.06.2007, Artvin Sacinka)

Mythimna vitellina (Hubner, 1808) (24.06.2007, Artvin Sacinka)

Sub-family Bryophilinae Guenée (1837)

Bryophila muralis (Forster, 1771) (18.07.2009, Artvin Seyitler)

Sub-family Amphipyrinae Ochsenheimer (1816)

Amphipyra pyramidea (Linneaus, 1758) (23.06.2007, Murgul)

Family Nolidae Hampson (1894)

Sub-family Chloephorinae (Stainton, 1859)

Nycteola asiatica (Krulikovsky, 1904) (06.07.2008, Artvin Kafkasor)

Family Pter ophoridae

Sub-family Pterophorinae (Zeller, 1841)

Pterophorus pentadactyla (Linnaeus, 1758) (07.06.2008, Artvin Kafkasor)

Family Lymantriidae Hampson (1893)

Sub-family Calliterinae Butler (1881)

Calliteara pudibunda (Linnaeus, 1758) (19.05.2008 and 09.06.2008, Murgul)

Sub-family Orgyiinae Hubner (1819)

Euproctis chrysorrhoea (Linnaeus, 1758) (15.06.2008 and 29.06.2009, Yusufeli; 13.06.2008, Ardanuc)

Sub-family Lymantriinae Hubner (1819)

Lymantria dispar (Linnaeus, 1758) (13.06.2008 and 22.06.2009, Ardanuc; 29.06.2007, Savsat)

Lymantria monacha (Linnaeus, 1758) (22.06.2009, Ardanuc; 14.06.2008 and 23.06.2009, Savsat)

Sub-family Arctorninae Linnaeus (1758)

Leucoma salicis (Linnaeus, 1758) (28.06.2007, Ardanuc; 30.06.2007 and 15.06.2008, Yusufeli)

species number and ratio of the families are shown in Fig. 2 while the species identified in Artvin region, the dates and locations are shown in Table 2.

The highest number of species was in the family Lycaenidae (61) followed by Satryridae (27), Nymphalidae (25) and Hesperiidae (20), respectively. The lowest number of species was in the family Zygaenide, Saturniidae, Nolidae and Pterophoridae (1) (Fig. 2). The highest number of species was found in grassland, forest and agricultural habitat types, respectively. The lowest number of species was found in urban habitat types. The

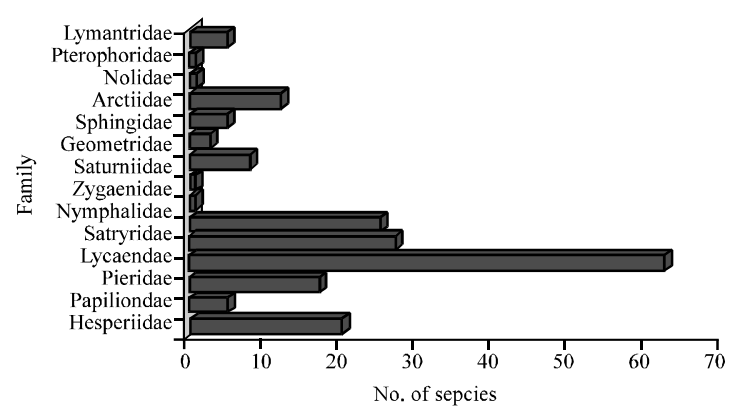

Fig. 2: The species number and ratio of the families 
highest number of species was catched in Yusufeli (102), followed by Artvin (50), Ardanuc (49), Hatila National Park (35), Savsat (22) and Murgul (22). The lowest number of species was catched in Arhavi (9) and Hopa (13) (Fig. 3 and 4). Most of Noctudae species was catched by light trap. Light trap was used only Hatila National Park and Murgul district.

In this research, 192 species belonging to 1 families of sub-order 339 were captured and identified in Artvin region of Black sea. Lepidopteran species were collected from different habitat types (agricultural crops to conifer or deciduous forests), locations (city to mountain) and time periods (May through September) to perform the best sampling of Artvin province. Artvin has very high ecosystem diversity in both forest and agricultural lands which may increase insect species richness (including Lepidoptera). The number of species was the highest for forest and the lowest for urban habitats.

There was significant difference between these two habitat types in terms of species richness. Forested habitats had a higher species richness than that of agricultural habitats. Most of species had a wide altitudinal distribution from $250-1800 \mathrm{~m}$. These results suggest that the increasing plant diversity may affect species richness positively. Increased niche diversity provided by trees in forest should lead to increased insect species richness because trees are larger and more complex in their architecture and live longer than herbaceous plants (Lawton, 1978). The highest number of species was collected during summer months with a peak in July and decreasing through autumn. The same trend was also observed for the number of specimens. The highest number of specimens was collected in July and June. The lowest number of specimens was collected in May. The peak activity period for Lepidopterans in Artvin province was between June and August. This can be related to the phenology of plants and changing environmental conditions of ecosystems.

The period of peak Lepidopteran numbers is an indicator of optimum abiotic and biotic conditions in the research areas. Changes in the structural complexity of plants over time might also affect the number of Lepidopteran species and individuals. The highest number of species was collected in Yusufeli. These results suggest that the increasing plant diversity may affect species richness positively. Increased niche diversity provided by trees in forest should lead to increased insect species richness because trees are larger and more complex in their architecture and live longer than herbaceous plants (Lawton, 1978).

Artvin province has got a rich flora and fauna. In this rich fauna, the abundance of variety of Lepidoptera has

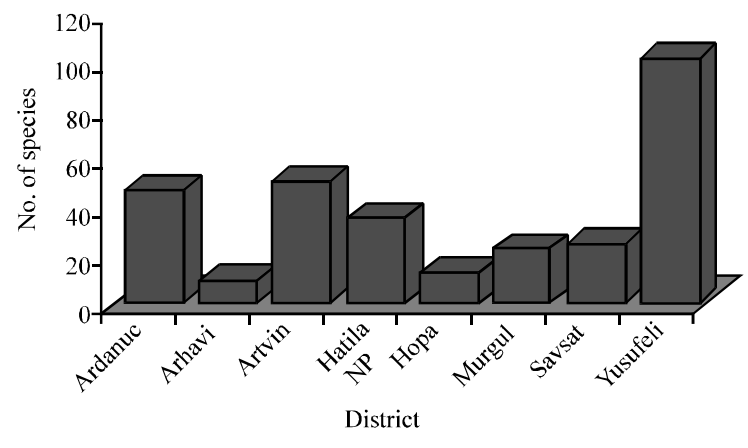

Fig. 3: Number of species in Artvin's localities

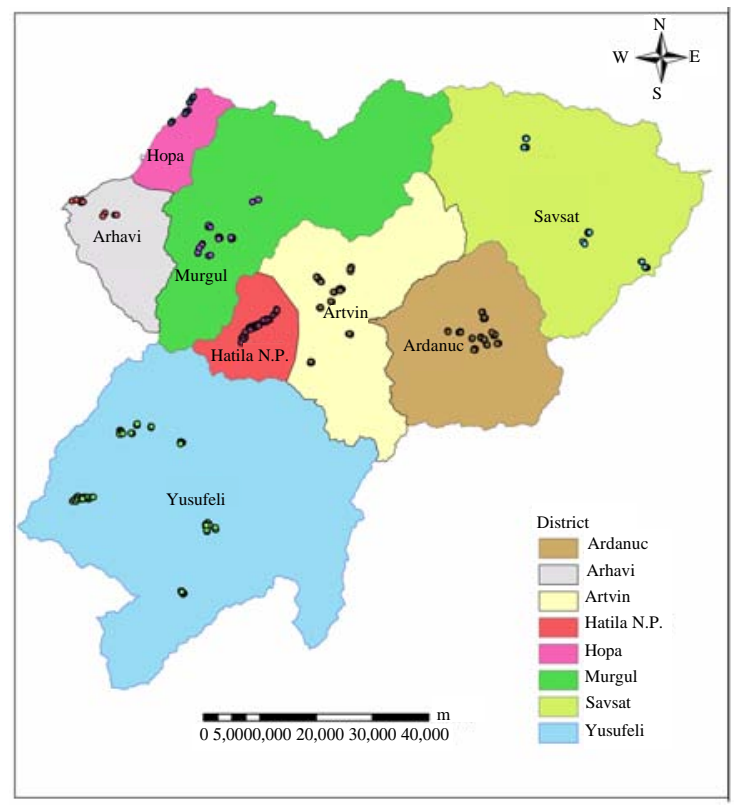

Fig. 4: Distribution of species

formerly taken the attention of many observers. Vegetation type is not reported for all species in this article because some species were found in every vegetation type which would make the article much longer. According to host plants, Papilio machaon, Leptidea sinapis, Pieris rapae, Pieris brassicae, Agrius convolvuli and Agrotis saucia are harmful insects to different agricultural plants.

Total 25 butterfly species (Satyrium ilicis, Satyrium acaciae, Satyrium w-album, Favonius quercus, Celastrina argiolus, Polygonia c-album, Saturnia pyri, Hydriomena impluviata, Alcis repandata, Campaea margaritata, Campaea margaritata, Laothoe populi, Arctia villica, Callimorpha quadripunctaria, Hyphantria cunea, Autographa gamma, Catocala elocata, Catocala nupta, Agrotis c-nigrum, Amphipyra pyramidea, Calliteara pudibunda, Euproctis 
chrysorrhoea, Lymantria dispar, Lymantria monacha, Leucoma salicis) identified as a result of different studies have formed some of the harmful insects causing harm to different forest trees and fruit trees. Larvae of other species eat grasses and forage crops ( $\mathrm{Mol}$ and Avci, 1997; Arslan, 1998; Oymen, 1990; Ozkazanc, 1998; Kaygin et al., 2009).

In this study, some of the species identified have been noted as harmful or potentially harmful for forests. These are A. villica, A. gamma, E. chrysorrhoea, $L$. dispar, L. salicis. The conservation status of plants and animals is one of the most widely used indicators for assessing the condition of ecosystems and their biodiversity. At the global scale, the best source of information on the conservation status of plants and animals is the IUCN red list of threatened species (IUCN, 2009). IUCN red list has got 8 Lepidoptera species in Near Threataned (NT). They are Carcharodus flocciferus, Carcharodus lavatherae, Chazara brisei, Hipparchia statilinus, Polyommatus damon, Polyommatus dorylas, Polyommatus eros and Pseudophilotes vicrama. Others species are identified as Least Concern (LC), Not Applicaple (NA) and Data Deficient (DD).

\section{CONCLUSION}

Climate is a major factor determining the distribution of species as well as the distribution of the vegetation. Climate change may simply shift these distributions but for a number of reasons, plants and animals may not be able to keep track of these changes (Schweiger et al., 2008). Climate change is already impacting some populations and is likely to affect additional species more significantly in the future (Settele et al., 2008). Within woodlands, many butterfly species rely on open areas, clearings, grass patches or woodland margins and require regular forest management (Van Swaay and Warren, 1999). A major factor in the decline of such species is the widespread changes in woodland management across Artvin, leading to reduced habitat suitability (Konvicka et al., 2008). Pesticides and herbicides kill butterflies. Furthermore, domestic and agricultural pollution (such as nitrogen deposition) leads to a faster succession of vegetation thus reducing the area of suitable habitat and habitat connectivity substantially. Have declared that there are 345 species in Turkey while 192 species were identified in Artvin in this study. This study indicates that Lepidoptera diversity is quite high in Artvin.

\section{REFERENCES}

Akbulut, S., B. Yuksel and A. Keten, 2003. The Lepidoptera (Insecta) Fauna of Duzce Province, Turkey. Turk. J. Zool., 27: 257-268.

Andow, D.A., 1991. Vegetational diversity and arthropod population response. Ann. Rev. Entomol., 36: $561-586$.

Arslan, Y., 1998. Insect species damaging on elm, common alder, maple and willow in Bartin Province. M.Sc. Thesis, Zonguldak Karaelmas University, Bartin.

Beskardes, V., 2002. The butterfly and moth (Lepidoptera) species living in Istanbul Catalca administration forests. M.Sc. Thesis, Istanbul University, Forestry Faculty, Istanbul.

Borror, D.J., C.A. Triplehorn and N.F. Johnson, 1989. An Introduction to the Study of Insects. 6th Edn., Saunders College Publishing. Fort Worth, Philadelphia, San Diego, pp: 875.

Can, F., 2008. The geometrid moths (Lepidoptera) from the Middle and Eastern black sea Regions of Turkey. Turk J. Zool., 32: 351-358.

Canakcioglu, H., 1993. Collecting-preparation-preserving and identification of insects. Forestry Faculty, Istanbul University, Istanbul, Turkey. Publication No. 3768, pp: 616.

Cassie, B., 2007. A World of Butterflies. 1st Edn., Chanticleer Press, Inc., New York.

Covell, C.V., 1984. A Field Guide to the moths of Eastern North America. Houghton Mifflin, Boston, Massachusetts, ISBN-13: 9780395260562 , pp: 496.

Eminagaoglu, O. and F.E. Bak, 2008. Dendroflora of Artvin. Proceedings of the International Science Conferences of the South Colchis Biodiversity, Batumi, Georgia. Funet.

Gillott, C., 2005. Entomology. Springer, Dordrecht, The Netherlands, pp: 831.

Groombridge, B., 1992. Global Biodiversity: Status of the Earth's Living Resources. Chapman and Hall, London, UK., pp: 614.

Hakyemez, A., 1994. Noctuidae (Lep.) species in forests of Zonguldak regional directorate. Rev. Faculty Forestry Unive. Istanbul Ser. A, 44: 111-133.

Higgins, L.G., 1982. Butterflies of Britain and Europe. Harper Collins Publishers, London.

IUCN, 2009. 2009 IUCN Red List of Threatened Species. IUCN, Cambridge, UK.

Kansu, A., 1963. The list for Turkish Lepidoptera: V. Plant Prot. Bull., 3: 208-223.

Kaygin, A.T., Z. Yildiz and M. Avci, 2009. Lepidoptera fauna in Bartin province, in Western black sea Region of Turkey. Afr. J. Agric. Res., 4: 815-822. 
Konvicka, M., J. Benes, O. Cizek, F. Kopecek, O. Konvicka and L. Vitaz, 2008. How too much care kills species: Grassland reserves, agri-environmental schemes and extinction of Colias myrmidone (Lepidoptera: Pieridae) from its former stronghold. J. Insect Conserv., 12: 519-525.

Lawton, J.H., 1978. Host-Plant Influences on Insects Diversity: The Effects of Space and Time. In: Diversity of Insect Faunas, Mound, L.A. and N. Waloff (Eds.). Blackwell Scientific Publications, Oxford, UK., pp: 105-125.

Mol, T. and M. Avci, 1997. Some Sphingidae species in Marmara Region. Rev. Faculty Forestry Univ. Istanbul, Ser. A, 47: 15-29.

Mol, T., 1977. Gometridae species living in Marmara and Eagean Regional Forests. Rev. Faculty Forestry Univ. Istanbul, 2329: 125-125.

Okyar, Z. and N. Aktac, 1999. Faunistic and taxonomic studies on the Geometridae species of Turkish Thrace. Turk. J. Zool., 23: 99-132.

Okyar, Z. and N. Aktac, 2006. Identification of butterfly (Lepidoptera: Rhopalocera) fauna of Gok ̃̃ßeada And Bozcaada, Turkey. Pak. J. Biol. Sci., 9: 76-79.

Oymen, T., 1990. Important lepidoptera species damaging on coniferous species in Turkey. Rev. Faculty Forestry Univ. Istanbul, Ser. B, 40: 59-66.
Ozkazanc, N.K., 1998. Insects damage on oak, beech and hornbeam trees in Bartin area forests. M.Sc. Thesis, Zonguldak Karaelmas University, Bartin.

Pedigo, L.P., 1996. Entomology and Pest Management. 2nd Edn., Iowa State University Press, USA., pp: 679.

Romoser, S.W. and G.J. Stoffolano, 1994. The Science of Entomology. William C Brown Publishers, Iowa, USA., pp: 532.

Rossing, W.A.H., H.M. Poehling and G. Burgio, 2003. Landscape management for functional biodiversity. IOBC/WPRS Bull., 26: 1-220.

Schweiger, O., J. Settele, O. Kudrna, S. Klotz and I. Kuhn, 2008. Climate change can cause spatial mismatch of trophically interacting species. Ecology, 89: 3472-3479.

Settele, J., O. Kudrna, A. Harpke, I. Kuhn and C. Swaay et al., 2008. Climatic risk atlas of European butterflies. Biorisk, 1: 1-710.

Shields, O., 1989. World number of butterflies. J. Lepidopterists Soc., 43: 178-183.

Van Swaay, C. and M. Warren, 1999. Red Data Book of European Butterflies (Rhopalocera). Nature and Environment, No. 99, Council of Europe Publishing, Strasbourg. 